BUHEP-06-02

INT-PUB 06-04

hep-ph/0602229

\title{
Linear Confinement and AdS/QCD
}

\author{
Andreas Karch ${ }^{1}$, Emanuel Katz ${ }^{2}$, Dam T. Son ${ }^{3}$, Mikhail A. Stephanov ${ }^{4}$ \\ 1 Department of Physics, University of Washington, Seattle, WA 98195 \\ 2 Department of Physics, Boston University, Boston, MA 02215 \\ 3 Institute for Nuclear Theory, University of Washington, Seattle, WA 98195 \\ 4 Department of Physics, University of Illinois, Chicago, IL 60607 \\ E-mail: karch@phys.washington.edu, amikatz@bu.edu, \\ son@phys.washington.edu, misha@uic.edu
}

\begin{abstract}
In a theory with linear confinement, such as QCD, the masses squared $m_{n, S}^{2}$ of mesons with high spin $S$ or high radial excitation number $n$ are expected, from semiclassical arguments, to grow linearly with $S$ and $n$. We show that this behavior can be reproduced within a putative 5-dimensional theory holographically dual to QCD (AdS/QCD). With the assumption that such a dual theory exists and describes highly excited mesons as well, we show that asymptotically linear $m^{2}$ spectrum translates into a strong constraint on the infrared behavior of that theory. In the simplest model which obeys such a constraint we find $m_{n, S}^{2} \sim(n+S)$.
\end{abstract}




\section{Introduction}

Over the recent years it has become clear that gauge/gravity correspondence [1] can be used to extract information about four-dimensional strongly coupled gauge theories by mapping them onto gravitational theories in five dimensions. The term AdS/QCD is often used to describe the efforts to apply a five-dimensional theory on an anti-de Sitter (AdS) gravity background to learn something about QCD [2-17]. Although for QCD the exact form of the gravity dual is not yet known, there are two complementary approaches to the problem. One is to start from a string theory, choosing the background in such a way as to reproduce such essential ingredients of QCD as confinement [3] or matter in the fundamental representation [4], and study the resulting QCD-like theories. Another, bottom-up, approach is to begin with QCD and attempt to determine or constrain the dual theory properties by matching them to known properties of QCD using gauge/gravity correspondence. From a practical point of view, one can model experimental data surprisingly well [12-14] by a local effective theory on a cutoff AdS space (AdS "slice"). The ultraviolet (UV) conformal invariance of QCD (due to asymptotic freedom) is matched by the conformal isometry of the AdS background of the dual $5 \mathrm{~d}$ theory, while confinement, in the simplest realization, is modeled by a hard wall cutting off AdS space in the infrared (IR) region, as first introduced in [5]. The bottom-up approach is related to an attempt undertaken by A.A. Migdal in the 1970's [18] to determine the meson spectrum by imposing the requirement of conformal invariance on QCD two-point correlators and using the Padé approximation, as well as to the open-moose models based on an infinite number of hidden local symmetries [19], as discussed in [20].

One criticism that has been brought against this program is that it so far appeared to be unable to describe correctly either (radially) excited rho mesons or higher spin mesons $[21,22]$. The meson spectrum in AdS/QCD is determined by solving for the eigenmodes of a $5 \mathrm{~d}$ gauge field living on the cutoff AdS. With the simplest cutoff - the hard IR wall - the spectrum of squared masses $m_{n}^{2}$ is similar to that of a Schrödinger equation for a particle in a box, i.e., for high excitation number, $n \gg 1, m_{n}^{2}$ grow as $n^{2}$.

On the other hand, data shows growth consistent with $m_{n}^{2} \sim n$, see, e.g., Fig. 1] and [24]. Furthermore, for large $n$ a heuristic semiclassical argument in favor of the behavior $m_{n}^{2} \sim n$ for QCD can be given [22]. The highly excited mesons can be thought of as an ultrarelativistic quark-antiquark pair executing the semiclassical motion in a potential growing linearly with the separation (due to the confining flux tube). With the typical momentum and energy of the quark motion related to the mass of the meson as $p=E=m_{n} / 2$ and the energy related to the typical separation $L$ (the size of the meson) as $E=\sigma L$, where $\sigma$ is the confining string (chromoelectric flux tube) tension, the typical size of an excited meson is

$$
L \sim m_{n} / \sigma .
$$

On the other hand, semiclassical Bohr-Sommerfeld quantization requires $\int p d x \sim n$, 


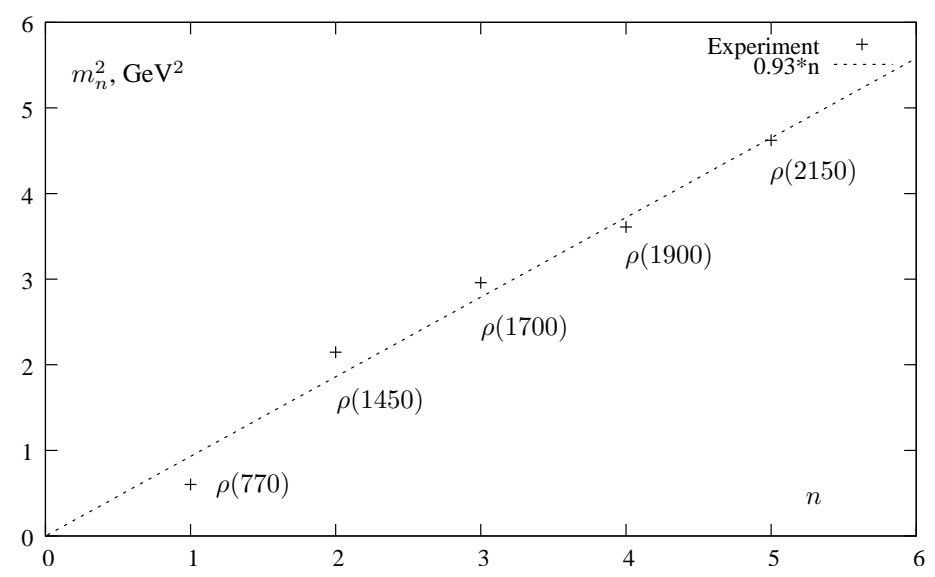

Figure 1: The squared masses of the first few $\rho$ resonances versus their consecutive number $n$ [23]. The straight line is the fit $m_{n}^{2} \sim n$.

which means

$$
m_{n}^{2} \sim \sigma n
$$

Such a behavior is also observed in the $1+1$ dimensional 't Hooft model [25] where linear confinement can be demonstrated analytically.

A similar situation occurs for high spin $S \gg 1$. The well-known argument based on a picture of a high spin meson as a semiclassically rotating relativistic open string the confining flux tube - predicts Regge behavior $m_{S}^{2}=2 \pi \sigma S$. However, as was shown in [14], in that case one finds a very similar problem in the hard wall model: while one can nicely match the experimental properties of the first few resonances, the growth of the squared masses with spin $S$ is $m_{S}^{2} \sim S^{2}$ as opposed to the expected Regge behavior.

The purpose of this note is to point out that the asymptotic behavior of the spectrum of highly excited mesons $m_{n}^{2} \sim n^{2}$ is by no means an intrinsic property of AdS/QCD. We wish to emphasize that, contrary to thus far rather common assumption, the spectrum of the highly excited mesons is not determined by the ultraviolet behavior of the AdS/QCD (which is already constrained to be asymptotically AdS). Rather, it crucially depends on the details of the infrared region. That this must be so can be clearly seen by recalling that the size $L$ of the mesons grows with their excitation number (II).

Below we shall give an explicit example of the IR wall that gives the desired

$$
m_{n}^{2} \sim n \quad \text { and } \quad m_{S}^{2} \sim S
$$

growth of the masses at large $n$ and $S$. At this point, we can give no explicit example of a background in an ab initio string theory that behaves in the way that we propose. However, since the growth (3) is a generic property of any linearly confining gauge theory, we may formulate our result as an IR constraint on the holographic dual of any such theory, including QCD. We shall speculate briefly about how such an IR behavior could arise from tachyon condensation in string theory. 


\section{Background geometry and overview}

The gravitational backgrounds we are interested in can still be thought of as cutoff AdS spaces, but instead of the hard-wall IR cutoff we shall look at spacetimes that smoothly cap off. The only background fields we are considering are the dilaton $\Phi$ and the metric $g_{M N}$. The mesons are described by $5 \mathrm{~d}$ fields propagating on this background with the action given by

$$
I=\int d^{5} x \sqrt{g} e^{-\Phi} \mathcal{L}
$$

where $\mathcal{L}$ is the Lagrangian density and $g=\left|\operatorname{det} g_{M N}\right|$. We shall begin by considering a generic background parameterized by two functions $A(z)$ and $\Phi(z)$ such that:

$$
\begin{aligned}
& g_{M N} d x^{M} d x^{N}=e^{2 A(z)}\left(d z^{2}+\eta_{\mu \nu} d x^{\mu} d x^{\nu}\right) \\
& \Phi=\Phi(z)
\end{aligned}
$$

where $\eta_{\mu \nu}=\operatorname{diag}(-1,1,1,1)$. We shall then determine the conditions that the background $A(z)$ and $\Phi(z)$ should obey to reproduce the Regge-like behavior of the mass spectrum Eq. (3). By considering the spectrum of radial $\rho$ excitations only we conclude that the linear combination $\Phi-A$ must behave as $z^{2}$ at large $z$ to agree with Eq. (2). In addition, conformal symmetry in the UV demands that $\Phi-A \sim \log z$ at small $z$. The simplest solution to both these constraints is $\Phi-A=z^{2}+\log z$. It has the advantage that the spectrum of excited $\rho$ masses can be determined exactly: $m_{n}^{2}=4(n+1)$.

In order to determine $A$ and $\Phi$ functions separately we then consider higher spin mesons. We find that the behavior as in (3) requires that the metric function $A$ does not have any contribution growing as $z^{2}$ at large $z$. In the simplest case obeying this constraint, $A=-\log z, \Phi=z^{2}$, the spectrum can be found exactly: $m_{n, S}^{2}=4(n+S)$.

\section{Rho mesons}

We shall work under the assumption that there exists a local effective action on this background that is dual to QCD, i.e., terms with a higher number of derivatives have to be suppressed. An incomplete justification of this assumption together with a discussion of the associated limitations can be found in Section 6. For the vector and axial meson sector the simplest action one can write down containing up to 2 derivatives is the $\mathrm{SU}\left(N_{F}\right)_{L} \times \mathrm{SU}\left(N_{F}\right)_{R}$ gauge field action with a bifundamental scalar whose vacuum expectation value is responsible for both the explicit and the spontaneous chiral symmetry breaking. This is the picture advertised in $[12,13]$ and it directly implements the ideas of [4] regarding how flavor is included in AdS/CFT as gauge fields living on the worldvolume of flavor branes. The analogy with [4] also fixes the coupling to the background dilaton to be an overall $e^{-\Phi}$ as expected from a D-brane. The gauge coupling $g_{5}$ is fixed by matching the UV asymptotics of current-current two-point function 
between bulk and boundary theories $[12,19]$. The action at quadratic order in the fields and derivatives reads

$$
I=\int d^{5} x e^{-\Phi(z)} \sqrt{g}\left\{-|D X|^{2}+3|X|^{2}-\frac{1}{4 g_{5}^{2}}\left(F_{L}^{2}+F_{R}^{2}\right)\right\}
$$

with $g_{5}^{2}=12 \pi^{2} / N_{c}$. The boundary condition on the gauge fields $A_{L}$ and $A_{R}$ at $z=0$ as required by the holographic correspondence, is given by the value of the sources of the currents $J_{L}$ and $J_{R}$ in 4 d theory. The IR boundary condition now, in the case of the smooth wall extending to $z=\infty$, is simply that the action is finite. The ambiguity of the choice of the IR boundary condition in a theory with hard wall [12] is not present in the theory with the smooth wall cutoff.

To determine the spectrum of the $\rho$ mesons we need only the quadratic part of the action for the vector-like gauge field $V=A_{L}+A_{R}$. We use the gauge invariance of the action to go to the axial gauge $V_{z}=0$ [12]. The equation for the 4 d-transverse components $V_{\mu}^{T}\left(\partial^{\mu} V_{\mu}^{T}=0\right)$ has normalizable solutions, $v_{n}$, only for discrete values of 4 d momentum $q^{2}$ equal to $m_{n}^{2}$ :

$$
\partial_{z}\left(e^{-B} \partial_{z} v_{n}\right)+m_{n}^{2} e^{-B} v_{n}=0
$$

where $B=\Phi(z)-A(z)$. Via the substitution

$$
v_{n}=e^{B / 2} \psi_{n}
$$

this equation can be brought into the form of a Schrödinger equation

$$
\begin{gathered}
-\psi_{n}^{\prime \prime}+V(z) \psi_{n}=m_{n}^{2} \psi_{n}, \\
V(z)=\frac{1}{4}\left(B^{\prime}\right)^{2}-\frac{1}{2} B^{\prime \prime} .
\end{gathered}
$$

In the particular case of $B=\Phi-A=z^{2}+\log z$, we have $V=z^{2}+3 /\left(4 z^{2}\right)$ and the Schrödinger equation (10) is exactly solvable. More generally, for the quantum mechanical system ${ }^{1}$

$$
-\psi^{\prime \prime}+\left[z^{2}+\frac{m^{2}-1 / 4}{z^{2}}\right] \psi=E \psi
$$

the eigenvalues are $(n=0,1,2, \ldots)$

$$
E=4 n+2 m+2
$$

and the corresponding normalized eigenfunctions are

$$
\psi_{n}(z)=e^{-\frac{z^{2}}{2}} z^{m+1 / 2} \sqrt{\frac{2 n !}{(m+n) !}} L_{n}^{m}\left(z^{2}\right) .
$$

\footnotetext{
${ }^{1}$ For integer values of $m$ Eq. (12) can be viewed as a radial equation for a two-dimensional harmonic oscillator with orbital momentum $m$.
} 
where $L_{n}^{m}$ are associated Laguerre polynomials. The $\rho$ meson mode equation (10) is of this form with $m=1$. We can easily read off the squared masses of the $\rho$ s from this:

$$
m_{n}^{2}=4(n+1) \text {. }
$$

The scale of the masses is fixed by the coefficient of the $z^{2}$ term in $B$, which violates explicitly the scale invariance. ${ }^{2}$ Matching to Eq. (2) we conclude that this coefficient is proportional to the QCD string tension $\sigma$. We measure masses in units in which this coefficient is equal to 1 .

Undoing the change of variables (9), we get the original mode functions $v_{n}=$ $e^{z^{2} / 2} \sqrt{z} \psi_{n}$, hence

$$
v_{n}(z)=z^{2} \sqrt{\frac{2 n !}{(1+n) !}} L_{n}^{1}\left(z^{2}\right) .
$$

From the analytic form of the wavefunction we also can read off the corresponding decay constants [12]:

$$
F_{\rho_{n}}^{2}=\frac{1}{g_{5}^{2}}\left[v_{n}^{\prime \prime}(0)\right]^{2}=\frac{8(n+1)}{g_{5}^{2}},
$$

whose large $n$ behavior is also in accord with semiclassical QCD arguments [22]. It is interesting to note that, since $F_{\rho_{n}}^{2} / m_{n}^{2}=2 / g_{5}^{2}$ is $n$-independent, our simplest choice of the background $B=z^{2}+\log z$ reproduces the ad hoc resonance model of duality discussed in [26].

Note that in order to get the correct $m_{n}^{2} \sim n$ behavior for large $n$ it was crucial that the analog Schrödinger potential describes essentially a harmonic oscillator at large $z$. This is easy to see applying the WKB approximation for large $n$. The distance between successive levels $m_{n}^{2}$ of the Schrödinger equation (10) is given by the frequency of the classical oscillation in the potential $V$ :

$$
\frac{d m_{n}^{2}}{d n}=\pi\left[\int_{z_{1}}^{z_{2}} \frac{d z}{\sqrt{m_{n}^{2}-V(z)}}\right]^{-1},
$$

where $z_{1,2}$ are the turning points. For large $m_{n}$, i.e., large $z_{2}$, and $z_{1} \rightarrow 0$, the integral is dominated by large $z \sim z_{2} \sim m_{n}$. This matches the expected growth of the size of the highly excited mesons in QCD $-L \sim m_{n}$ (see Eq. (II) $)^{3}$

By choosing a different function $V(z)$ (i.e., a different background $B(z)$ ) one can adjust the constant $O(1)$ term in $m_{n}^{2}$, but as long as $V(z) \sim z^{2}$ for large $z$, the spectrum will remain equidistant at large $n .^{4}$

\footnotetext{
${ }^{2}$ In the theory with hard IR wall this role was played by the position of the wall $z_{m}$.

${ }^{3}$ The proper definition of the meson size should be based on the meson formfactor, which is determined by cubic terms in the action. In this paper we only consider quadratic part of the action and indirectly infer the size of the meson from the extent of its wave function in the 5 -th dimension.

${ }^{4}$ One can also notice that the choice of $B \sim-z^{2}$ leads to the same asymptotics of $V(z)$ as the choice $B \sim z^{2}$ we made. The former, however, is physically unacceptable because it leads to an $m_{n}^{2}=0$ solution to the $\rho$ mode equation (8).
} 
Matching only the spectrum of the $\rho$ mesons we are only able to constrain the linear combination $B=\Phi-A$ of the dilaton and the metrics background functions $\Phi$ and $A$. In the next section we shall see that all the $z^{2}$ asymptotics must all be in $\Phi$ and none in $A$.

\section{$4 \quad$ Higher spin mesons}

In order to create higher spin mesons we need to act with a higher spin current on the vacuum. Just like for the vector mesons, on the gravity side, we have to introduce a higher spin field whose normalizable modes determine meson masses and decay constants. In the free theory in the far UV the corresponding current becomes a conserved twist 2 current, so the higher spin field has to become a massless higher spin field whose equations of motion are uniquely fixed by gauge and coordinate invariance [27].

In the full theory the higher spin field will have to acquire a mass in the IR by a generalization of the Higgs mechanism. As long as the mass of the higher spin field remains finite in the IR, it is easy to see that it will not affect the highly excited modes. We will discuss this phenomenon in detail in the next section in the special case of the spin $S=1$ axial vectors. For our discussion of higher spin mesons we will only consider massless higher spin fields in the $5 \mathrm{~d}$ bulk.

Since we will only be concerned with the spectrum of the higher spin mesons, we shall not consider the full $5 \mathrm{~d}$ action, but only its quadratic (free) part. It is known that simultaneously gauge and general-coordinate invariant action can be written for a higher spin field in a space with vanishing Weyl tensor (the part of the Riemann tensor which does not affect the Ricci tensor) [28-30]. The background we consider (5) is conformally flat and obeys this condition. Thus we proceed on assumption that such action does exist.

The gauge field of spin $S$ is represented by a tensor $\phi_{M_{1} \ldots M_{S}}$ of rank $S$ totally symmetric over its indices. As discussed above, we require the action to be invariant with respect to the gauge transformation with gauge parameter $\xi_{M_{2} \ldots M_{S}}$ itself a symmetric rank $S-1$ tensor:

$$
\delta \phi_{M_{1} \ldots M_{S}}=\nabla_{\left(M_{1}\right.} \xi_{\left.M_{2} \ldots M_{S}\right)},
$$

where $\nabla$ is (general coordinate) covariant derivative and parentheses denote index symmetrization. ${ }^{5}$ The quadratic part of the gauge and coordinate invariant action for this field must have the form

$$
I=\frac{1}{2} \int d^{5} x \sqrt{g} e^{-\Phi}\left\{\nabla_{N} \phi_{M_{1} \ldots M_{S}} \nabla^{N} \phi^{M_{1} \ldots M_{S}}+M^{2}(z) \phi_{M_{1} \ldots M_{S}} \phi^{M_{1} \ldots M_{S}}+\ldots\right\}
$$

where the omitted terms are similar to the two written out but with coordinate indices contracted in alternative ways. The mass coefficient $M^{2}(z)$ would be zero for flat space,

\footnotetext{
${ }^{5}$ It is known that gauge invariance can be imposed at most for a restricted class of gauge transformations with traceless $\xi^{N}{ }_{N} \ldots=0$ gauge parameter. This fact will not play a role in our discussion.
} 
but must be nonzero otherwise to cancel terms arising from commutation of covariant derivatives to ensure gauge invariance of $I$. For the pure AdS space $M^{2}(z)$ is a constant $M^{2}=S^{2}-S-4$. Using the method outlined below we can find $M^{2}(z)$ for a general metric (5), however, as we shall see, the same method gives the mode equation directly, bypassing $M^{2}(z)$.

Firstly, similar to the $S=1$ case (Section 3), we utilize the gauge invariance to go over to the axial gauge $\phi_{z \ldots}=0$. In this gauge, the part of the action involving the transverse and traceless part of the field $\phi\left(\partial_{\mu} \phi^{\mu} \ldots=0\right.$ and $\left.\phi^{\nu}{ }_{\nu \ldots}=0\right)$ decouples. It is also easy to see that this part of the action only involves the terms explicitly written out in (20), while the omitted terms do not contribute.

Secondly, we use the fact that the axial gauge still allows residual gauge transformations obeying $\xi_{z \ldots}=0$ and

$$
\delta \phi_{z \ldots}=\nabla_{z} \xi_{\ldots}+\nabla_{\left(. \xi_{\ldots) z}\right.}=\xi_{\ldots}^{\prime}-2(S-1) A^{\prime} \xi_{\ldots}=0 .
$$

This can be easily integrated to find that the $z$ dependence of the residual gauge parameter $\xi$ is given by

$$
\xi_{\mu_{2} \ldots \mu_{S}}\left(z, x^{\mu}\right)=e^{2(S-1) A(z)} \tilde{\xi}_{\mu_{2} \ldots \mu_{S}}\left(x^{\mu}\right) .
$$

The action (20) for transverse traceless modes must be invariant under the gauge transformations with parameter (22). As in [14] this requirement is easiest to implement working in terms of a rescaled higher spin field $\tilde{\phi}$ defined by

$$
\phi_{\ldots}=e^{2(S-1) A} \tilde{\phi} \ldots
$$

This field simply shifts by a value independent of $z$ under gauge transformations (22), $\delta \tilde{\phi}_{\ldots}=\partial_{\left(. \tilde{\xi}_{\ldots)}\right.}$. Therefore the action written in terms of $\tilde{\phi}$ should contain only derivatives of $\tilde{\phi}$. Thus if we are to substitute (23) into action (20) we must find (discarding appropriate boundary terms)

$$
I=\frac{1}{2} \int d^{5} x e^{5 A} e^{-\Phi}\left\{e^{4(S-1) A} e^{-2 A(1+S)} \partial_{N} \tilde{\phi}_{\mu_{1} \ldots \mu_{S}} \partial_{N} \tilde{\phi}_{\mu_{1} \ldots \mu_{S}}\right\}
$$

The equation for the modes $\tilde{\phi}_{n}$ of the transverse traceless field $\tilde{\phi}_{\text {... }}$ can be now easily derived from the action (24):

$$
\partial_{z}\left(e^{(2 S-1) A} e^{-\Phi} \partial_{z} \tilde{\phi}_{n}\right)+m_{n}^{2} e^{(2 S-1) A} e^{-\Phi} \tilde{\phi}_{n}=0
$$

which has the form (8) with $B=\Phi-(2 S-1) A$.

Converting to Schrödinger form using the procedure from Section 3 we see that the only way to have the slope $d m_{n}^{2} / d n$ independent of $S$ is to keep all $z^{2}$ asymptotics in $\Phi$ and none in $A$. For $A=-\log z$ and $\Phi=z^{2}$ the Schrödinger potential reads

$$
V(z)=z^{2}+2(S-1)+\frac{S^{2}-1 / 4}{z^{2}}
$$


This has the same form as the potential in Eq. (12). The eigenvalues corresponding to the squared masses of the mesons now can easily be read off using (13)

$$
m_{n, S}^{2}=4(n+S)
$$

which generalizes our result (15) to higher $S$.

We can also read off the UV conformal dimension of the operators $\mathcal{O} \cdots$ in QCD dual to the higher spin field $\phi \ldots$. Since the rescaled field $\tilde{\phi}$ allows a solution going to a constant at $z=0$ boundary, its boundary value should be identified with the source of the operator $\mathcal{O} \cdots$. The scaling dimension of the field $\tilde{\phi}$ is $\left[\tilde{\phi}_{\ldots}.\right]=\left[\phi_{\ldots .}\right]-2(S-1)=2-S$ (in units in which $[z]=-1$ ). Thus $\left[\mathcal{O}^{\cdots}\right]=4-\left[\tilde{\phi}_{\ldots}\right]=2+S$, i.e., the twist is indeed equal to 2 .

\section{The axial sector}

Similar to the $\rho$ mesons, the axial vector meson $a_{1}$ masses and decay constants can be obtained from the modes of the axial gauge field $A=\frac{1}{2}\left(A_{L}-A_{R}\right)$ in the bulk. Unlike the vector sector however the axial field picks up a $z$-dependent 5 d mass via the Higgs mechanism from the background scalar $X$ that encodes the chiral symmetry breaking [12]. The axial vector meson mode equation, which follows from Eq. (7), reads:

$$
\partial_{z}\left(e^{-\Phi(z)} e^{A(z)} \partial_{z} a_{n}\right)+\left[m_{n}^{2}-g_{5}^{2} e^{2 A(z)} X(z)^{2}\right] e^{-\Phi(z)} e^{A(z)} a_{n}(z)=0 .
$$

The linearized equation of motion for the field $X$ reads:

$$
\partial_{z}\left(e^{-\Phi(z)} e^{3 A(z)} \partial_{z} X(z)\right)+3 e^{-\Phi(z)} e^{5 A(z)} X(z)=0 .
$$

We are looking for a solution with asymptotic form

$$
X(z) \stackrel{z \rightarrow 0}{\rightarrow} \frac{1}{2} M z+\frac{1}{2} \Sigma z^{3} .
$$

around $z=0$ (in the UV), where the coefficient $M$ is the UV $(z=0)$ boundary condition given by the quark mass matrix, while the coefficient $\Sigma$ - the chiral condensate

- is determined dynamically by the boundary condition in the IR. For large $z$ (in the IR) on the background $\Phi=z^{2}$ the equation for $X$ becomes

$$
X^{\prime \prime}-2 z X^{\prime}+\frac{3}{z^{2}} X=0 \quad(z \gg 1)
$$

Two linearly independent solutions of this equation have asymptotics $e^{z^{2}} \rightarrow \infty$ and $\exp \left\{-(3 / 4) z^{-2}\right\} \rightarrow 1$. Since the equation is linear, selecting one of the solutions in the IR (the $X<\infty$ one, of course) gives $\Sigma$ simply proportional to $M$. This is not 
what one wants in a theory with spontaneous symmetry breaking such as QCD. It is clear that one has to consider higher order terms in the potential $U(X, \ldots)$ for $X$ and all other scalar condensates. Such a potential would introduce nonlinearity in the equation (29) for $X$ and consequently in the relation between $\Sigma$ and $M$. In addition, one expects higher order derivative terms to become important in determining the precise background at intermediate values of $z$.

For a generic $U(X, \ldots)$ there will be a solution approaching a constant as $z \rightarrow \infty$. For such a solution, as $z \rightarrow \infty$, the term $X^{\prime \prime}$ becomes negligible (together with all other higher derivatives) compared to $-2 z X^{\prime}$, and equation behaves as a 1st order, rather than a 2 nd order equation. That means only one parameter family of solutions exist in the IR region (that parameter being the value $X(\infty)$ ). Continuing each such solution into the UV we find corresponding value for $M$ and $\Sigma$, therefore determining (parametrically) the function $\Sigma(M)$, which is nonlinear for generic nonlinear $U(X, \ldots)$.

We conclude that the spectrum in the axial sector depends sensitively on the precise form of the $5 \mathrm{~d}$ potential $U(X, \ldots)$, as well as other higher order terms. However, we expect that in the IR $X$ will have a solution that goes to a constant as $z \rightarrow \infty$. The constant value that $X$ approaches gives the IR value of the mass of the axial gauge field. As can be seen from the axial mode equation (28), the contribution from finite $X$ is suppressed by a factor $e^{2 A}=z^{-2}$. Therefore, the large $z$ asymptotics of the Schrödinger equation potential $V(z)$ will be the same as for the $\rho$ mesons. Hence, the slope of the $a_{1}$ radial excitation trajectory will be the same as the slope for the $\rho$-mesons $d m_{n}^{2} / d n=4$ as expected from semiclassical arguments in the Introduction.

As we mentioned in the previous section, the higher spin mesons find themselves in a similar situation. We know that the corresponding currents will not be conserved in the interacting theory, so the dual higher spin field has to pick up a mass in the IR and hence our analysis based on massless higher spin fields will not be valid. However, as long as the mass of the higher spin field remains finite in the IR, at large $z$ and hence at large $n$ the mass term can be neglected and our result

$$
m_{n, S}^{2} \sim 4(n+S)
$$

is reliable for large $n$ and $S$.

\section{Conclusions and discussion}

In this paper we demonstrated that under the assumption of a local $5 \mathrm{~d}$ bulk description of QCD there is a smoothing of the IR wall (asymptotically unique) that gives the right large $n$ and large $S$ behavior for highly radially or orbitally excited mesons characteristic of linear confinement $m_{n, S}^{2} \sim(n+S)$. We have found that such a spectrum can be achieved in a nontrivial dilaton background $\Phi$ in (4) with the following large $z$ (IR) asymptotics: $\Phi \sim z^{2}$. We have also found that the metric background function 
$A$ in (44) cannot have a $z^{2}$ contribution at large $z$ if the slope of the radial excitation trajectories $d m_{n, S}^{2} / d n$ is to be the same for all $S$.

It is interesting to observe that in such a dilaton/metric background the slopes of $n$ and $S$ trajectories automatically coincide:

$$
\frac{d m_{n, S}^{2}}{d n}=\frac{d m_{n, S}^{2}}{d S} .
$$

This matches the expectation from QCD if one considers highly excited mesons as semiclassically oscillating $(n \gg 1)$ or rotating $(S \gg 1)$ strings - the confining flux tubes connecting quark and antiquark. Indeed, the frequencies of the classical oscillatory and rotational motions of the relativistic Nambu-Goto open string at the same energy coincide [31]. The poles of the Veneziano amplitude also correspond to the spectrum obeying (33) [24].

We also found the size of the mesons growing linearly with their mass as required by semiclassical arguments in QCD Eq. (11). In comparison, with hard IR wall at $z_{m}$, excited mesons would have all the same size $L \sim z_{m}$.

It is worth pointing out that the semiclassical arguments which picture highly excited mesons as excited confining flux tubes apply in the limit of large $N_{c}$ when the string breaking is suppressed. In the dual theory, the meson coupling $g_{5}^{2}=12 \pi^{2} / N_{c}$ is small in this limit, making resonances narrow and allowing us to neglect loop corrections to the action (7).

One interesting phenomenological aspect of the quadratic dilaton is that the coupling $g_{5}^{2} e^{\Phi}$ becomes strong in the IR. Modes of excitation number $n$ explore $z$ values of order $\sqrt{n}$. Thus their self-coupling grows as $e^{z^{2}} \sim e^{n}$. For any given excitation number $n$ the modes will be weakly coupled for sufficiently large $N_{c}: g_{n n n} \sim e^{n} / N_{c}$. This then suggests that for a fixed large $N_{c}$ the meson resonances will become strongly coupled not at $n \sim N_{c}$ but already at $n \sim \log \left(N_{c}\right)$.

Let us close with a detailed discussion of the assumptions we made and the limitations of this line of thought. Our approach relies on the proposition that QCD can be described holographically by a local $5 \mathrm{~d}$ action, that is, the terms with a higher number of derivatives are suppressed. In this local description all higher spin fields have to be included as elementary fields. One should think of this as being the spacetime action corresponding to string field theory where all excitations of the string get explicitly incorporated as spacetime fields.

Since in QCD there is no parametric separation between the string tension and the mass gap, the dual gravity will have curvatures of order the string scale and hence there will be no scale separation between the massless and massive string modes; it is hence very natural to include them all on an equal footing. However, it appears unjustified from this point of view to expect the action of those fields to be dominated by the low derivative terms, that is, to be local. Without separation of scales, it is not clear what is suppressing the higher derivative terms. And without that knowledge, it is not clear how to improve systematically on the approximations made. 
Nevertheless, our assumption that such a local action is sensible from the stringtheory point of view might be supported by a theorem proposed in [32], stating that at large $N_{c}$ the action should always be local ${ }^{6}$. If this claim is correct, it would be the large $N_{c}$ that is suppressing the non-local terms, quite contrary to standard AdS/CFT intuition.

A conservative point of view on the 5d Lagrangian would be to consider it as a phenomenologically driven approach, along the line of Refs. [12,13], intelligently interpolating between the low-energy and high-energy limits of QCD. This approach, while being inspired by the AdS/CFT correspondence, may or may not have any direct relationship to the latter.

The background we found has an AdS geometry with a quadratic dilation $\Phi \sim z^{2}$ turned on. Obviously, one interesting question is how such a background would arise from a string theory. That is, what other fields have to be turned on in order to make this a good background for strings to propagate on? This would require us to construct the classical string theory on a spacetime with stringy curvature and presumably RRfluxes turned on. This type of background has been sought after now for over a decade without significant progress. While such a construction would obviously be desirable, our construction shows that as long as the assumption of locality holds many of the spacetime properties of such a theory can be reconstructed.

At least qualitatively we can see how the quadratic dilaton might arise. Confinement in the gauge theory is believed to be dual to closed string tachyon condensation in the bulk, so the simplest hypothesis is that in addition to the dilaton the only other field that is turned on is a closed string tachyon of a non-critical super string theory. It was shown in [34] that quite generically it is possible to engineer a closed string tachyon condensation process in which the only fields turned on are the dilaton and the tachyon, while all other fields, in particular the metric, can retain the background values. So it seems to be completely consistent in this framework to have an undisturbed background on top of which the closed string tachyon and the dilaton run. Of course from this point of view it is far from obvious why the dilaton profile should be quadratic. So in this respect our finding raises the interesting prospect that QCD data and/or semiclassical arguments using confining flux tubes can actually be used to gain new insights into the fascinating process of closed string tachyon condensation!

Last but not least, one might wonder why none of the known string theory duals to confining gauge theories exhibit the particular IR behavior we found. The point here is that up to this date the only $4 \mathrm{~d}$ examples of the gauge/string theory correspondence that are worked out have the string theory be well approximated by its low energy supergravity. On the gauge theory side this is reflected in a separation of scales -

\footnotetext{
${ }^{6}$ One can argue that as long as one is only interested in on-shell properties described by the quadratic part of the action, like the masses and decay constants, all higher derivative terms are essentially equivalent to the standard quadratic terms on a modified background [33]. However we want to claim that in the effective action fields of all spins propagate on the same background geometry, which would not be justified by the arguments in [33] alone.
} 
the string tension is much larger than the mass gap in the theory. Only the modes with masses above the mass gap but below the string tension are described by fields propagating on the gravity background. Note that even the confining examples in that class do not exhibit meson spectrum characteristic of the linear confinement. The squared meson masses in these theories grow as $n^{2}$ and not as $n$. Of course, at very high $n$, when the masses are much larger than the string tension one has to find linear growth in $n$. These modes however are stringy even in the known examples and will see a different background than the supergravity modes. Our results indicate that they will have to see the same exponential wall.

\section{Acknowledgments}

D.T.S. thanks the Institute for Advanced Study, where part of this work was completed, for hospitality. M.A.S. thanks the Institute for Nuclear Theory, University of Washington for hospitality. The work of A.K. was supported, in part, by the DOE under Contract No. DE-FG02-96-ER40956. The work of D.T.S. was supported, in part, by the DOE Grant No. DE-FG02-00ER41132 and by an IBM Einstein Endowed Fellowship from the Institute for Advanced Study. The work of M.A.S. was supported, in part, by DOE Grant No. DE-FG02-01ER41195 and the Alfred P. Sloan Foundation.

\section{References}

[1] J. M. Maldacena, Adv. Theor. Math. Phys. 2, 231 (1998).

[2] E. Witten, Adv. Theor. Math. Phys. 2, 505 (1998).

[3] J. Polchinski and M. J. Strassler, arXiv:hep-th/0003136.

[4] A. Karch and E. Katz, J. High Energy Phys. 06 (2002) 043.

[5] J. Polchinski and M. J. Strassler, Phys. Rev. Lett. 88, 031601 (2002).

[6] C. Csaki, H. Ooguri, Y. Oz, and J. Terning, J. High Energy Phys. 01 (1999) 017.

[7] L. Girardello, M. Petrini, M. Porrati, and A. Zaffaroni, Nucl. Phys. B 569, 451 (2000).

[8] J. Babington, J. Erdmenger, N. Evans, Z. Guralnik, and I. Kirsch, Phys. Rev. D 69, 066007 (2004).

[9] M. Kruczenski, D. Mateos, R. C. Myers, and D. J. Winters, J. High Energy Phys. 07 (2003) 049; 05 (2004) 041.

[10] H. Boschi-Filho and N. R. F. Braga, J. High Energy Phys. 05 (2003) 009.

[11] G. F. de Teramond and S. J. Brodsky, Phys. Rev. Lett. 94, 201601 (2005). 
[12] J. Erlich, E. Katz, D. T. Son, and M. A. Stephanov, Phys. Rev. Lett 95, 261602 (2005).

[13] L. Da Rold and A. Pomarol, Nucl. Phys. B 721, 79 (2005); J. High Energy Phys. 01 (2006) 157.

[14] E. Katz, A. Lewandowski, and M. D. Schwartz, arXiv:hep-ph/0510388.

[15] T. Sakai and S. Sugimoto, Prog. Theor. Phys. 113, 843 (2005); 114, 1083 (2006).

[16] J. Hirn and V. Sanz, J. High Energy Phys. 12 (2005) 030; J. Hirn, N. Rius and V. Sanz, Phys. Rev. D 73, 085005 (2006).

[17] S. Hong, S. Yoon and M. J. Strassler, J. High Energy Phys. 04 (2006) 003; arXiv:hep-ph/0501197.

[18] A. A. Migdal, Annals Phys. 109, 365 (1977); ibid 110, 46 (1978).

[19] D. T. Son and M. A. Stephanov, Phys. Rev. D 69, 065020 (2004).

[20] J. Erlich, G. D. Kribs, and I. Low, Phys. Rev. D 73, 096001 (2006).

[21] E. Schreiber, arXiv:hep-th/0403226.

[22] M. Shifman, arXiv:hep-ph/0507246.

[23] S. Eidelman et al. [Particle Data Group Collaboration], Phys. Lett. B 592, 1 (2004).

[24] P. D. B. Collins, An Introduction to Regge Theory and High-Energy Physics (Cambridge University Press, Cambridge, 1977).

[25] G. 't Hooft, Nucl. Phys. B75 (1974) 461.

[26] M. A. Shifman, arXiv:hep-ph/0009131.

[27] C. Fronsdal, Phys. Rev. D 18, 3624 (1978).

[28] C. Aragone and S. Deser, Phys. Lett. B 86, 161 (1979).

[29] C. Fronsdal, Phys. Rev. D 20, 848 (1979).

[30] T. Curtright, Phys. Lett. B 85, 219 (1979).

[31] See, e.g., B. Zwiebach, A First Course in String Theory (Cambridge University Press, Cambridge, 2004).

[32] E. Witten, "Spacetime reconstruction", talk given at JHS/60 conference, http://quark.caltech.edu/jhs60/witten/1.html. 
[33] J. Erlich, E. Katz, D. Son, and M. Stephanov, work in progress.

[34] H. Yang and B. Zwiebach, J. High Energy Phys. 08 (2005) 046; 09 (2005) 054. 